\title{
Synthesis of carboxymethyl starch grafted polyvinyl imidazole (CMS-g-PVIs) and their role as adsorbents for the removal of phenol
}

\author{
Fazal Haq ${ }^{1}$, Haojie Yu ${ }^{1+}$, Li Wang1, Lisong Teng ${ }^{2}$, Sahid Mehmood ${ }^{1}$, Muhammad Haroon ${ }^{1}$, \\ Bilal-Ul-Amin ${ }^{1}$, Shah Fahad ${ }^{1}$, Md Alim Uddin ${ }^{1}$, Di Shen ${ }^{1}$ \\ ${ }^{1}$ State Key Laboratory of Chemical Engineering, College of Chemical and Biological Engineering, Zhejiang University, Hangzhou 310027, P.R. China \\ ${ }^{2}$ Oncological Surgery and Cancer Center. The First Affiliated Hospital, Zhejiang University, 310003, P.R China
}

\begin{abstract}
Cigarette industry plays an important role in the economy of the advanced countries. But the cigarette smoke contains toxic chemicals such as phenol which causes various kind of diseases and affect human life. In this paper, we synthesized carboxymethyl starch grafted poly vinyl imidazole (CMS-g-PVIs) by reacting carboxymehtyl starch (CMS) with vinyl imidazole (VI). The structures of the CMS-g-PVIs were investigated by ${ }^{1} \mathrm{H}$ NMR and FT-IR. The crystalline properties of the CMS and CMS- $g$-PVIs were checked by XRD. The thermal properties of the original CMS and CMS-g-PVIs were investigated by TGA analysis. It was found that the modified starches had high thermal stability due to aromatic imidazole ring. The modified starches also showed more rough and distorted morphology as compared to native CMS. The CMS-g-PVIs were subjected for phenol adsorption and showed adsorption efficiencies of $0.170 \mathrm{~g} / \mathrm{g}, 0.190 \mathrm{~g} / \mathrm{g}, 0.192 \mathrm{~g} / \mathrm{g}$ and $0.199 \mathrm{~g} / \mathrm{g}$ for CMS, CMS-g-PVI 1, CMS-g-PVI 2 and CMS-g-PVI 3, respectively. Due to higher grafting ratio, CMS-g-PVI 3 showed good adsorption efficiency of $0.199 \mathrm{~g} / \mathrm{g}$ for phenol. The obtained results showed that the grafting of vinyl imidazole on CMS can increase the adsorption efficiency of native CMS towards phenol.
\end{abstract}

Keywords: Adsorption, Chemical modification, CMS-g-PVIs, Phenol

\section{Introduction}

Cigarette smoking is assumed to be a silent killer because the smoke contains numerous toxic gases. [1] According to survey 69 chemicals out of 7,000 chemicals are believed to be cancer causing [2-4]. Tobacco smoking was proved to be agent increasing suicidal behavior and suicide [2, 5-11]. Phenol increases the risk of different diseases especially, cancer, muscle weakness, convulsion and coordinate impairment [12-15]. In literature, different adsorbents were used for the removal of phenol. Chen et al. [16] used successfully carbon spherules and coal activated carbon for the removal of phenol, p-chlorophenol and p-nitrophenol. The adsorption efficiency of carbon spherules was found to be 35.55 $\mathrm{mg} / \mathrm{g}, 39.5 \mathrm{mg} / \mathrm{g}$ and $47.75 \mathrm{mg} / \mathrm{g}$, respectively for phenol, $p$-chlorophenol and $p$-nitrophenol. Similarly, the adsorpton capacity on coal activated carbon was $37.68 \mathrm{mg} / \mathrm{g}, 46.3 \mathrm{mg} / \mathrm{g}$ and $56.38 \mathrm{mg} / \mathrm{g}$ for phenol, $p$-chlorophenol and $p$-nitrophenol. For the removal of phenol and its derivatives from aqeous media, fungus Pleurotus sajor cajo was used as a sorbent [17]. The maximum adsorption efficiences were found to be $0.95 \mathrm{mmol} / \mathrm{g}, 1.24 \mathrm{mmol} / \mathrm{g}, 1.47 \mathrm{mmol} / \mathrm{g}$ and $1.89 \mathrm{mmol} / \mathrm{g}$ for phenol, $o$-chlorophenol, $p$-chlorophenol and 2,4,6-trichlorophenol, respectively. Termoul et al. [18] used chemically activated olive stones as a sorbent for the adsorption of phenol and 4-chlorophenol. The maximum adsorption capacity was found to be $189 \mathrm{mg} / \mathrm{g}$ for phenol. The biosorption of phenol on dried activated sludge was investigated, and the results showed adsorption efficiency of $86.1 \mathrm{mg} / \mathrm{g}$ [19].

However, the adsorption efficiency needs more attention. Herein, we synthesized bio-based adsorbent carboxymethyl starch grafted poly vinyl imidazole (CMS-g-PVIs). It is biodegradable, environmentally friendly, cost effective and more efficient for phenol adsorption. The CMS has bunch of hydroxyl groups and carboxylic
This is an Open Access article distributed under the terms of the Creative Commons Attribution Non-Commercial License (http://creativecommons.org/licenses/by-nc/3.0/) which permits unrestricted non-commercial use, distribution, and reproduction in any medium, provided the original work is properly cited.

Copyright (C) 2021 Korean Society of Environmental Engineers
Received June 10, 2020 Accepted September 21, 2020

${ }^{\dagger}$ Corresponding author

Email: hjyu@zju.edu.cn

Tel: +86-571-8795-3200 Fax: +86-571-8795-1612

ORCID: 0000-0002-7405-7881 
group on its backbone. As these groups have high affinity for making hydrogen bonding with phenol. So, due to presence of these groups, CMS acted as good sorbent for the removal of phenol. The adsorption capacity of the CMS was further increased by grafting VI on CMS main chain. The grafting of VI on CMS backbone increased the density of imidazole ring. The nitrogen atoms in imidazole ring made hydrogen bonding with the hydroxyl group of the phenol. Similarly, the aromatic imidazole ring also contributed to phenol adsorption by making $\pi-\pi$ interactions with the aromatic benzene ring of phenol. So, the grafting of VI on CMS increased the adsorption capacity of CMS-g-PVIs for phenol.

\section{Experimental Description}

\subsection{Materials}

Ethanol (99.7\%), phenol (99.0\%) and methanol (99.5\%) were bought from Sinopharm Chemical Reagent Co. Ltd., China. Carboxymethyl starch (CMS) was purchased from Aladdin, China. 1-vinyl imidazole (VI) (99.0\%) and potassium persulfate (KPS) (99.0\%) were bought from J\&K Scientific Ltd. All the reagents were used without further purification.

\subsection{Synthesis of CMS-g-PVIs}

The CMS-g-PVIs were synthesized according to the method described in literature [20, 21]. Firstly, $1.0739 \mathrm{~g}$ (6.62 mmol) of CMS was put into three necked round bottom flask and added $40 \mathrm{~mL}$ of distilled water to dissolve it. The reaction was kept under argon atmosphere at $60^{\circ} \mathrm{C}$ for $0.5 \mathrm{~h}$. Next, $178.9 \mathrm{mg}$ (0.66 mmol) of KPS was dissolved in $10 \mathrm{~mL}$ of distilled water and the KPS solution was added dropwise into the reaction mixture. Then run the reaction at same reaction conditions for $0.5 \mathrm{~h}$. Afterwards, $5.3 \mathrm{~mL}(59.5$ mmol) of vinyl imidazole was added to the reaction contents in drop-wise manner and reaction mixture was further stirred at 450 rpm for $3 \mathrm{~h}$. After $3 \mathrm{~h}$ the reaction was stopped and cool to room temperature. Afterward, the reaction contents were washed with mixture of methanol $(70 \mathrm{~mL})$ and water $(30 \mathrm{~mL})$ three times. Lastely, the product was washed with ethanol to remove the water and methanol traces. The product obtained after washing was placed in a vacuum oven at $40^{\circ} \mathrm{C}$ for $24 \mathrm{~h}$. Finally, the product was crushed into powder by pestle and mortar and stored.

\subsection{Characterization}

The ${ }^{1} \mathrm{H}$ NMR spectra were recorded on Bruker Advanx-400 DMX NMR spectrometer using $\mathrm{D}_{2} \mathrm{O}$ (basic). Nicolet 5700 IR spectrometer was used to record FT-IR spectra. The crystalline nature was checked on by using a XPert PRO ( $\mathrm{Cu} \mathrm{K} \alpha, \lambda=1.54 \AA$ ). Thermal properties were checked on TA-Q500 (Mettler-Toledo) under nitrogen atmosphere. The SEM images were taken on SU-8010 spectrometer (Oxford X-max80 instrument Hitachi Ltd). The Varian CARY 100 Bio UV/Visible spectrophotometer was used for the determination of phenol concentration.

\subsection{Adsorption Studies}

The adsorption affinity of the modified starches toward phenol was determined by conducting adsorption experiment.

\subsubsection{Filling of sample glass tubes}

For the filling of sample glass tubes five glass tubes were taken. First the filter from cigarette was cut by scissor into two equal halves. One half of the filter was inserted in the sample glass tube. Afterwards, specific quantity of CMS-g-PVIs was added in sample glass tube. The other half of the filter was then placed so that the sample was pressed in between the two halves. Four sample glass tubes were filled in the same manner, and one was loaded with blank cigarette filter.

\subsubsection{Phenol adsorption}

The assembly used for phenol adsorption experiment is shown in Fig. 1. The phenol source solution was prepared in two necked flask (c) by dissolving $29 \mathrm{~g}$ of phenol in a mixture of $50 \mathrm{~mL}$ of ethanol and $100 \mathrm{~mL}$ of distilled water. The two necked flask was set at $35^{\circ} \mathrm{C}$ in an oil bath. One neck of the two necked flask was linked to the gas flow meter (b) which intern connected to source of argon (Ar) gas (a). The second neck of the two necked flask was attached to the six sample glass tubes (d) connected to six conical flasks (e). Further attachment of the conical flasks (e) was made with conical flasks (f). The Ar gas was first blown via gas flow meter, and adjusted the speed of gas flow at $0.7 \mathrm{~m}^{3} / \mathrm{h}$. Then Ar gas was blown through the source solution of phenol where Ar gas obtained phenol as gaseous mixture of Ar-phenol. The gaseous Ar-phenol mixture was blown through the sample glass tubes. Four sample glass tubes were charged with $30.2 \mathrm{mg}$ of CMS, $30.2 \mathrm{mg}$ of CMS-g-PVI1, $30.1 \mathrm{mg}$ of CMS-g-PVI2 and $30.2 \mathrm{mg}$ of CMS-g-PVI3 and one was loaded with blank cigarette filter. In the sample glass tubes some quantity of phenol was sorbed by the sorbents. The remaining phenol was allowed into the ethanol $(75 \mathrm{~mL})$ containing conical flasks (e). The ethanol in conical flasks dissolved small

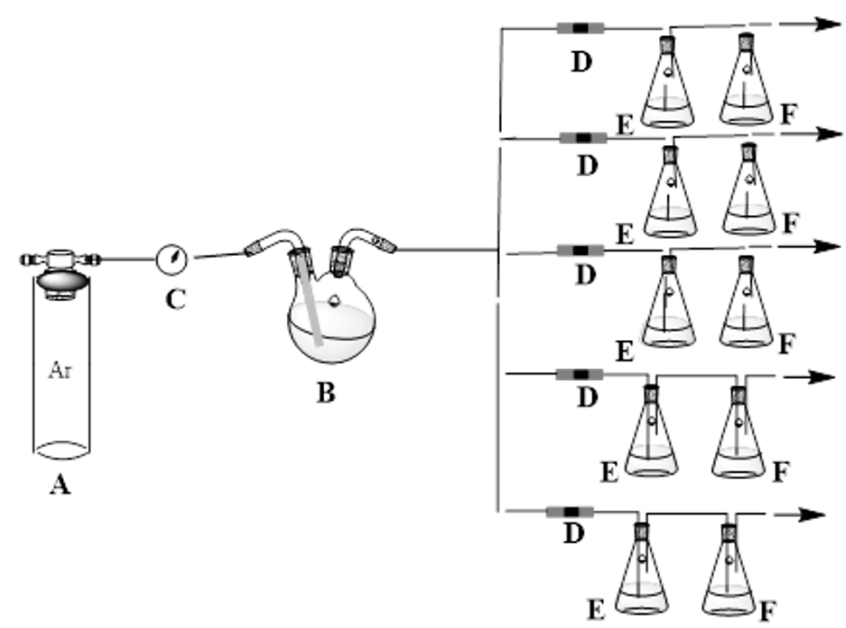

Fig. 1. Assembly used for the phenol adsorption experiment; (a) $\mathrm{Ar}$ gas cylinder, (c) gas flow meter (controlled the flow of gas at speed rate of $0.7 \mathrm{~m}^{3} / \mathrm{h}$ ), (b) two necked round bottom flask containing phenol source solution (29 g phenol, dissolved in $100 \mathrm{~mL}$ of water and $50 \mathrm{~mL}$ of ethanol), (d) sample glass tubes in which four tubes were filled with almost $30 \mathrm{mg}$ of adsorbent while one tube was filled with blank cigarette filter, (e) conical flasks containing $75 \mathrm{~mL}$ of ethanol and (f) conical flasks containing $75 \mathrm{~mL}$ of distilled water. 
amount of phenol. The Ar/phenol mixture was blown for $20 \mathrm{~min}$ through the sample glass tubes. To determine the phenol concentration, $2 \mathrm{~mL}$ of ethanol solution was taken by syringe from each conical flasks (e) and analyzed by UV-Vis spectrophotometer.

\section{Results and Discussion}

\subsection{Synthesis and Characterization of CMS-g-PVIs}

The CMS- $g$-PVIs were produced by free radical polymerization. This typical reaction was initiated by KPS. In this typical reaction CMS was treated with VI in water at $60^{\circ} \mathrm{C}$ for $4 \mathrm{~h}$. The synthetic reaction is shown Fig. 2 while the recipe details of CMS- $g$-PVIs are given in Table S1. The details of the structural characterization and other properties of the CMS-g-PVIs are discussed in their respective sections.

The ${ }^{1} \mathrm{H}$ NMR spectra of CMS-g-PVIs are shown in Fig. 3. The peak ranged from 5.2-5.4 ppm is for the proton of anomeric carbon labelled as C1a, b of the CMS. The bunch of peaks appeared in the region ranged from 3.0-4.3 ppm is for the protons of carbons C2-C7 of CMS and C9 of PVI, respectively. The peak allocated at the region ranged from 1.8-2.3 ppm is for the protons of C8 of the PVI constituent.

The emerged types of peaks appeared from 6.5-7.1 ppm are for the protons of $\mathrm{C} 10-\mathrm{C} 12$ of the imidazole ring present in the PVIs part. The appearance of all the characteristic peaks confirmed the synthesis of CMS-g-PVIs [22, 23]. The relative grafting ratios for CMS-g-PVIs were deliberated by using the following Eq. (1) [24].

$$
\text { Grafting ratio }=\frac{\text { area of } \mathrm{C}-8 \text { protons of } \mathrm{PVI} / 2}{\text { area of } \mathrm{C}-1 \mathrm{a}, \mathrm{b}, \mathrm{c} \text { of } \mathrm{CMS}}
$$

The relative grafting ratios for CMS-g-PVI1, CMS-g-PVI2 and CMS-g-PVI3 were found to be $0.49 \mathrm{~mol} / \mathrm{mol}, 0.56 \mathrm{~mol} / \mathrm{mol}$ and $0.84 \mathrm{~mol} / \mathrm{mol}$, respectively.

The variant functional groups present in structure of CMS-g-PVIs were confirmed by FT-IR. The bands in the IR spectra of CMS and CMS-g-PVIs are depicted in Fig. 4 (a). The bands appeared in the functional group region at $3,400,2,920$, and $1,610 \mathrm{~cm}^{-1}$ were assigned to the stretching vibration of $-\mathrm{OH},-\mathrm{CH}$ and $-\mathrm{C}=\mathrm{O}$ of the CMS. [20, 21] Similarly, in the functional region the band appeared at $3,109 \mathrm{~cm}^{-1}$ was attributed to stretching vibration of $\mathrm{C}=\mathrm{C}-\mathrm{H}$ and $\mathrm{N}=\mathrm{C}-\mathrm{H}$ of the imidazole ring in CMS-g-PVIs. The other characteristics bands appeared at 1,409 $\mathrm{cm}^{-1}, 1,546 \mathrm{~cm}^{-1} 1,643 \mathrm{~cm}^{-1}$ were ascribed to the $\mathrm{C}-\mathrm{H}$ bending and stretching vibration of $\mathrm{C}=\mathrm{C}$ and $\mathrm{C}=\mathrm{N}$ in vinyl imidazole ring [25]. Similarly, the bands appeared in the finger print region at $662 \mathrm{~cm}^{-1}$ and $1,081 \mathrm{~cm}^{-1}$ were assigned to $\mathrm{C}-\mathrm{N}$ vibration of azole ring and stretching vibration of -C-O-C-. The band appeared at $615 \mathrm{~cm}^{-1}$ was ascribed to the ring deformation out of plane bending. [26] The appearance of all these characteristics bands confirmed the successful grafting of VI on CMS backbone.

The XRD patterns of CMS and CMS-g-PVIs are depicted in Fig. 4 (b). The native CMS shows bunch of crystalline peaks at $2 \theta$ $=15.00^{\circ}, 17.03^{\circ}, 17.85^{\circ}, 22.78^{\circ}$ and $31.59^{\circ}$. The results are in accordance to the findings previously reported in literature. [24, 27] According to knowledge from the literature PVI also shows different peaks at $2 \theta=18^{\circ}, 22^{\circ}, 24^{\circ}, 30^{\circ}, 31^{\circ}, 36^{\circ}$ and $39^{\circ}$ [28]. These peaks were disappeared in CMS-g-PVI 1, CMS-g-PVI 2 and CMS-g-PVI

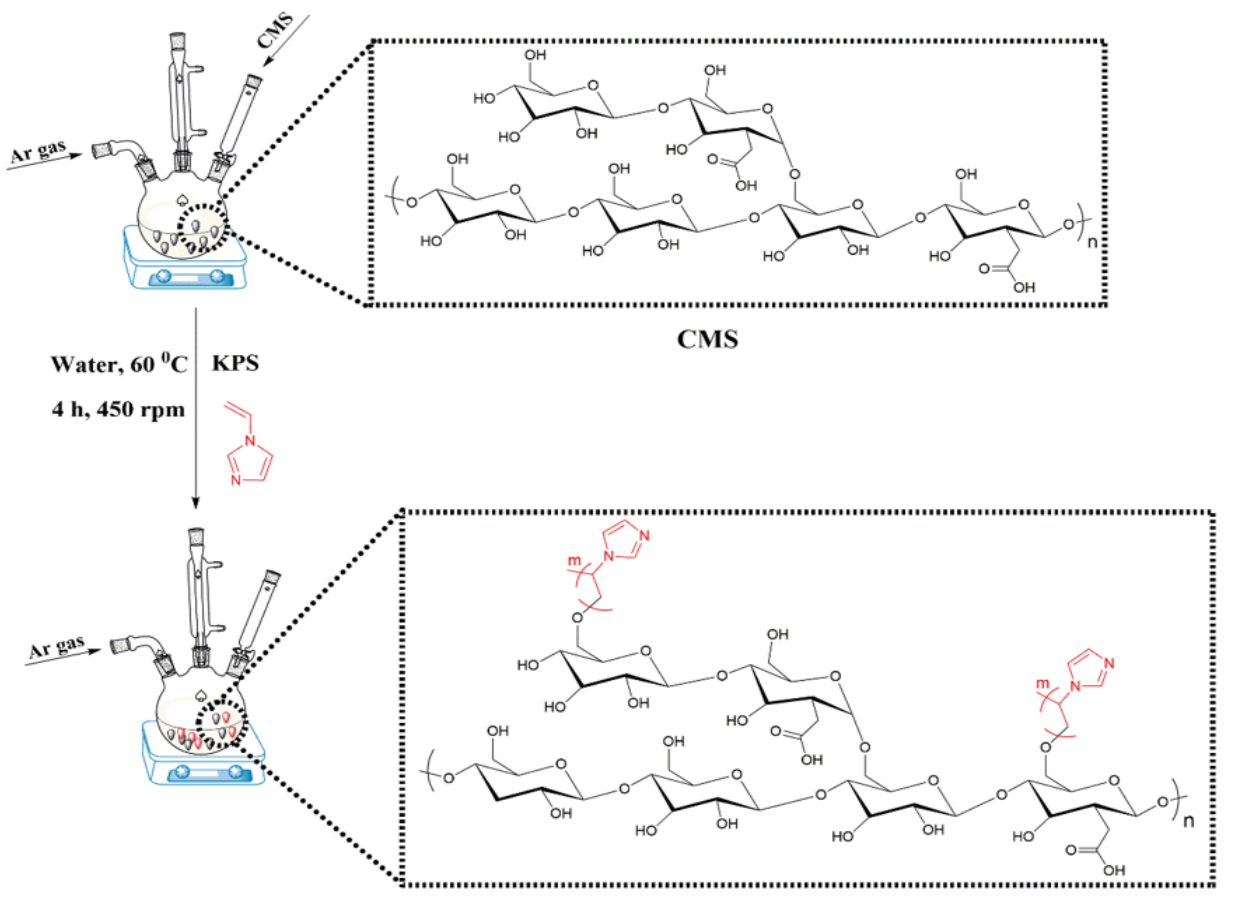

CMS-g-PVIs

Fig. 2. Synthesis of CMS-g-PVIs with experimental conditions of: CMS $1 \mathrm{~g}$, VI with variant mole ratio to CMS (CMS 1 and VI 3:6:9), KPS 1\% of both the monomers, water $50 \mathrm{~mL}$, stirring rate $450 \mathrm{rpm}$, inert atmosphere of $\mathrm{Ar}$ gas and temperature $60^{\circ} \mathrm{C}$. 
3 , respectively which showed the amorphous nature of these modified starches. The reason might be disrupting hydrogen bonding [29] between the hydroxyl groups present on CMS backbone. The disappearance of peaks in the XRD patterns of CMS-g-PVIs is also the evidence for their successful synthesis. The TGA and DTG analysis were done to explore the thermal behavior of CMS-g-PVIs. The TGA and DTG analysis results are shown in Fig. 5. CMS exhibited single step thermal decomposition at $\mathrm{DTG}_{\max } 294{ }^{\circ} \mathrm{C}$ due to decarboxylation and breakdown of main chain of AGU. This was in accordance to the range sited already in literature [20, 21].
Similarly, the CMS-g-PVI 1, CMS-g-PVI 2 and CMS-g-PVI 3, respectively also displayed a single step weight loss at $\mathrm{DTG}_{\max } 468$ ${ }^{\circ} \mathrm{C}, \mathrm{DTG}_{\max } 468{ }^{\circ} \mathrm{C}$ and $\mathrm{DTG}_{\max } 470{ }^{\circ} \mathrm{C}$ for the decomposition of imidazole ring. [30] So, after modification the thermal stability of the CMS-g-PVIs was increased. The increase in the thermal stability was caused by aromatic imidazole ring [23].

The CMS granules showed spherical and smooth surface as depicted in Fig. 6 (a) and (b). The Fig. 6 (c)-(h) showed the different surface morphologies for the copolymers CMS-g-PVI 1, CMS-g-PVI 2 and CMS-g-PVI 3, respectively. The obtained results showed

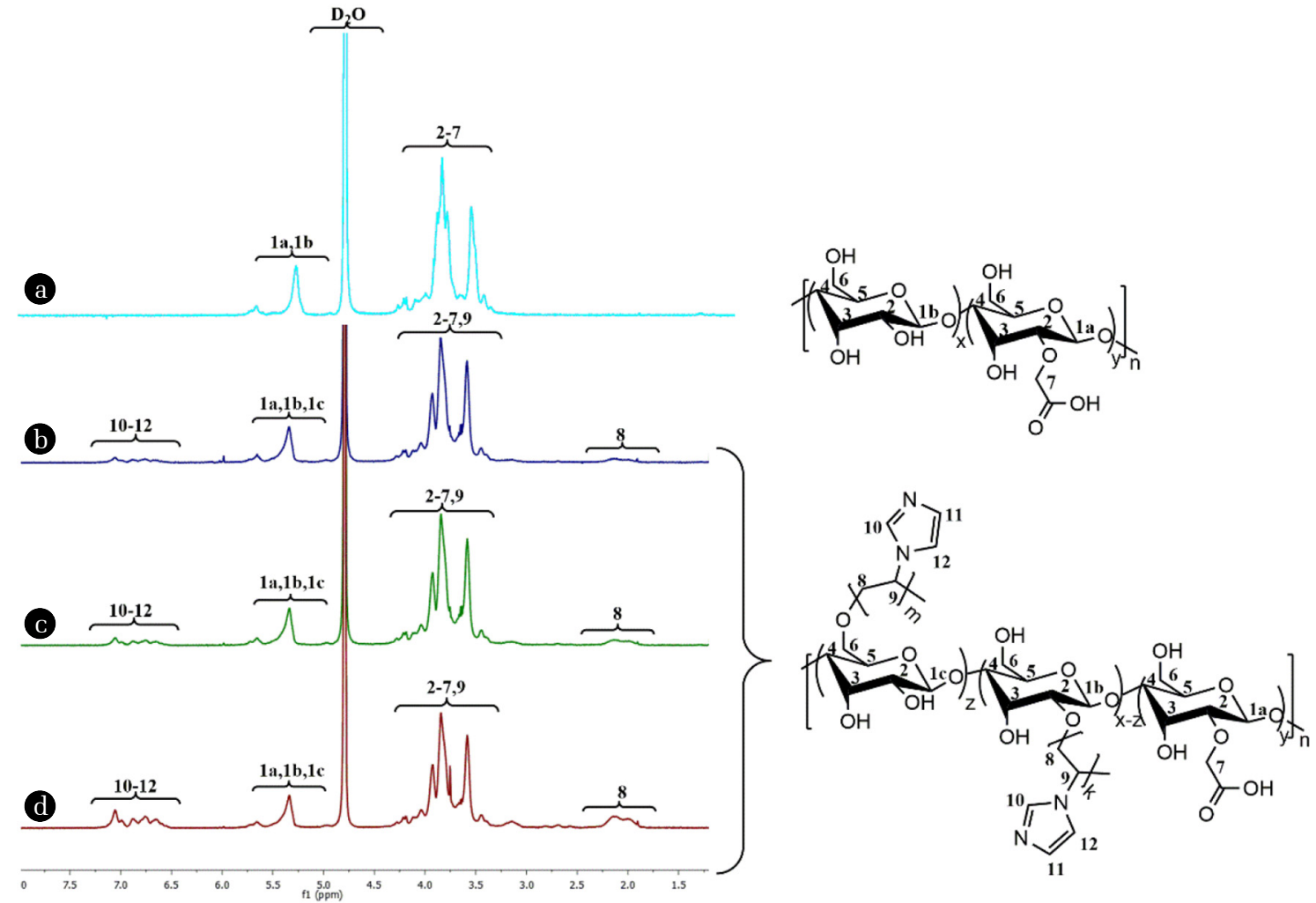

Fig. 3. ${ }^{1} \mathrm{H}$ NMR spectra of (a) CMS, (b) CMS-g-PVI 1, (c) CMS-g-PVI 2 and (d) CMS-g-PVI 3 in basic $\mathrm{D}_{2} \mathrm{O}$ at room temperature [22, 23].
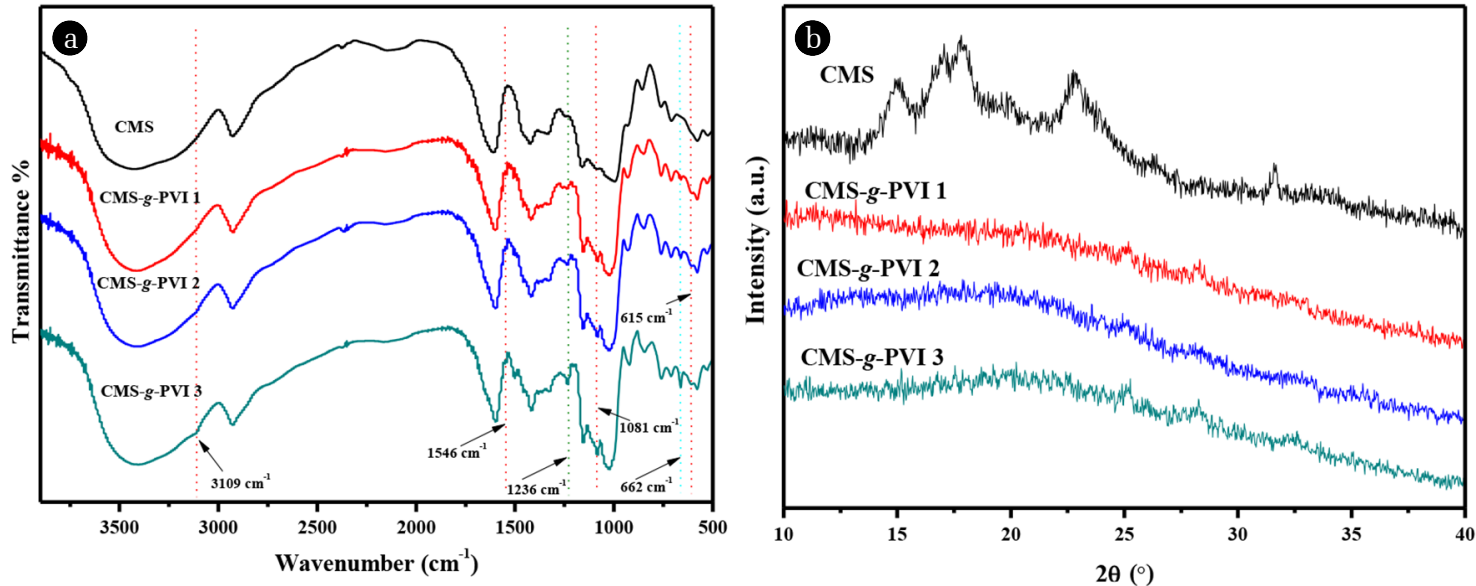

Fig. 4. (a) IR spectra and (b) XRD patterns of CMS, CMS-g-PVI 1, CMS-g-PVI 2 and CMS-g-PVI 3 at room temperature. 

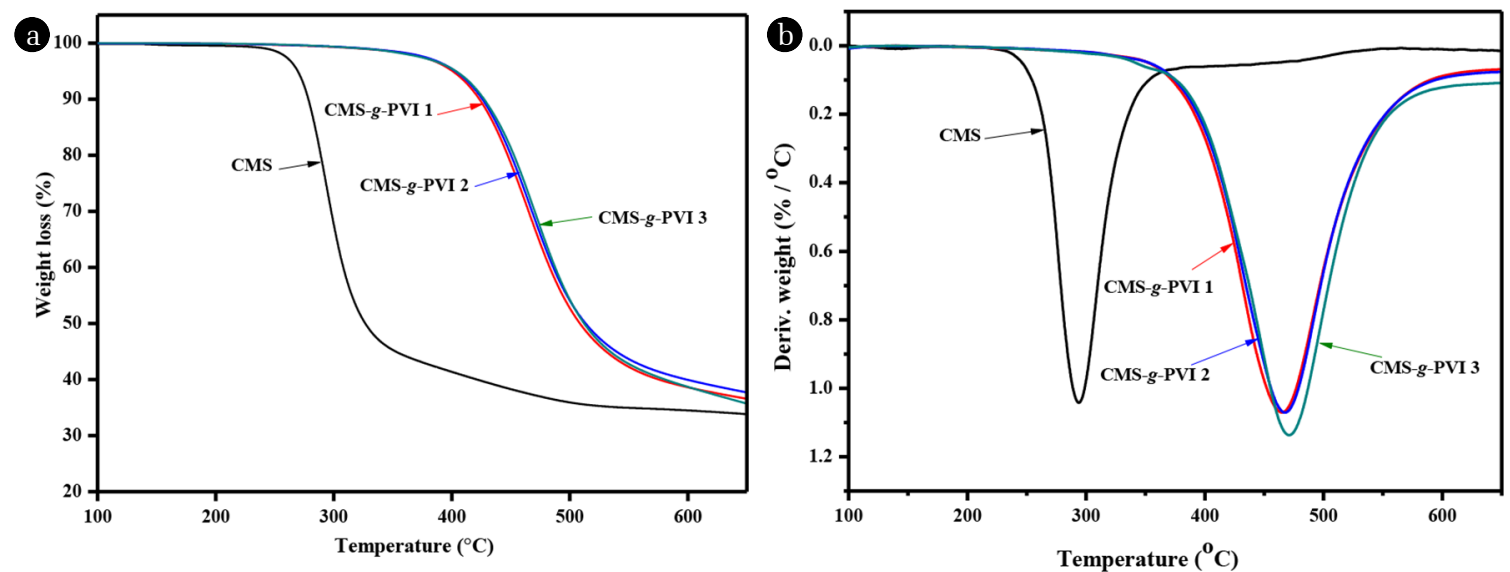

Fig. 5. (a) TGA and (b) DTG curves for CMS, CMS-g-PVI 1, CMS-g-PVI 2 and CMS-g-PVI 3 at the heating rate of $10^{\circ} \mathrm{C} / \mathrm{min}$ from $50^{\circ} \mathrm{C}$ to $600^{\circ} \mathrm{C}$ under the inert atmosphere of nitrogen.
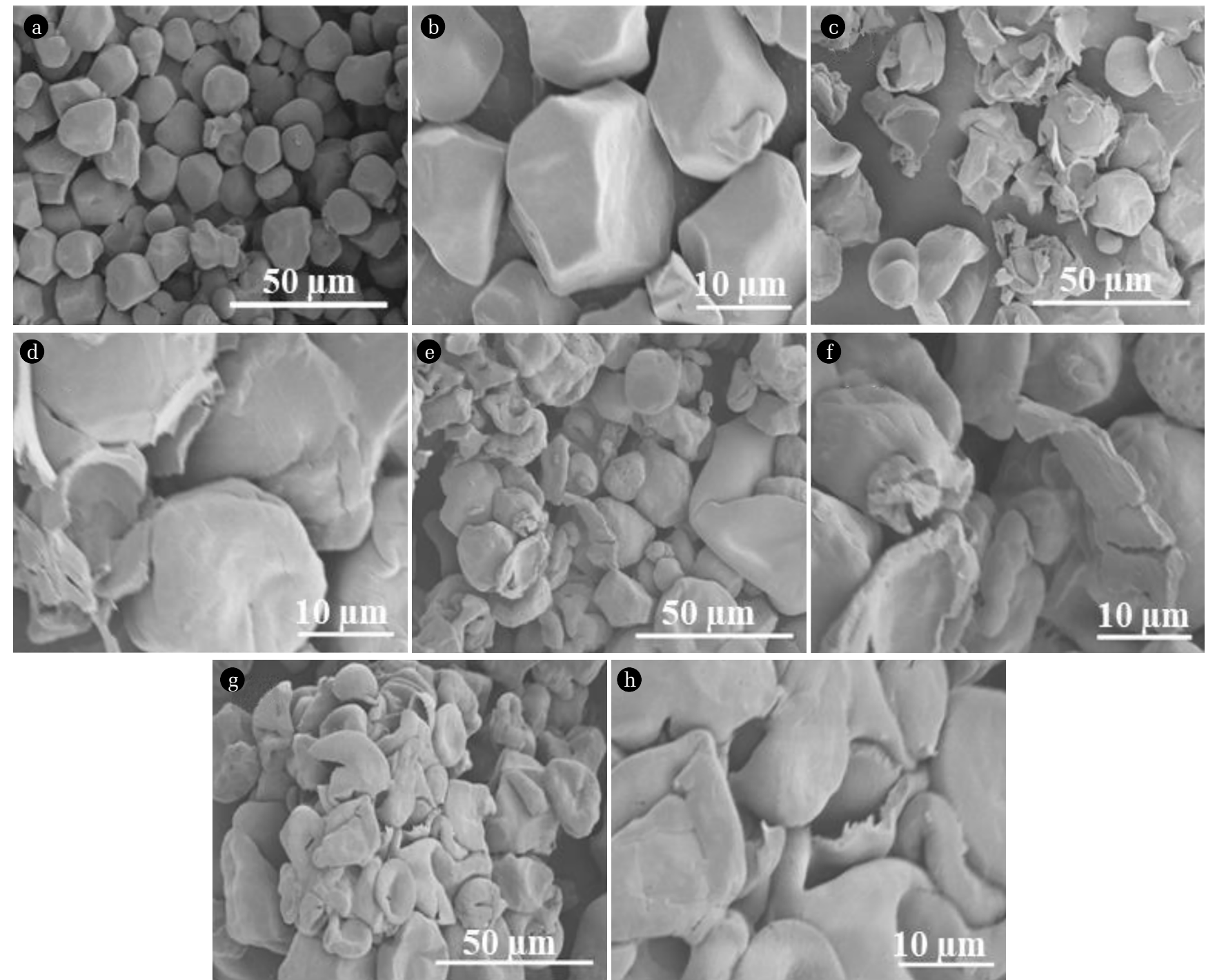

Fig. 6. SEM images of CMS (a) and (b), CMS-g-PVI 1 (c) and (d), CMS-g-PVI 2 (e) and (f) and CMS-g-PVI 3 (g) and (h). 
more rough surfaces for the CMS-g-PVI 1, CMS-g-PVI 2 and CMS-g-PVI 3. The rough surface is assumed to be more advantageous for the adsorption [24]. The Fig. 6 (g) and (h) showed more distorted surface for CMS-g-PVI 3 due to higher grafting ratio. The SEM images also provided an evidence for the successful synthesis of the CMS-g-PVIs.

\subsection{Adsorption Studies}

After the successful synthesis the CMS-g-PVIs were used as an adsorbent to their affinity towards phenol.

After passing Ar-phenol gaseous mixture through sample glass tubes containing adsorbent, the adsorbents adsorbed some amount of phenol.

The amount of phenol adsorbed by each particular adsorbent was calculated by subtracting the concentration of phenol in the

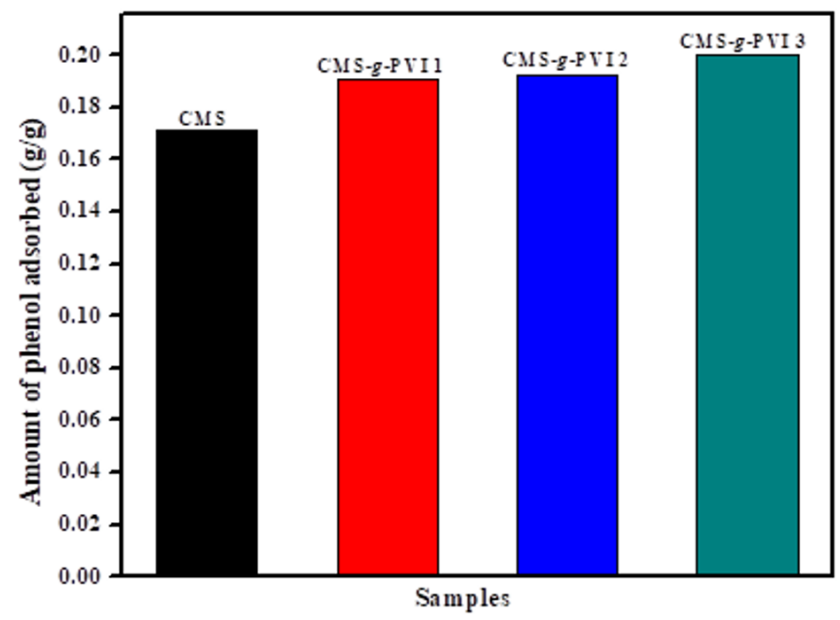

Fig. 7. Phenol adsorption affinities of CMS, CMS-g-PVI 1, CMS-g-PVI 2 and CMS-g-PVI 3 after 20 min flow of Ar-phenol gaseous stream at speed rate of $0.7 \mathrm{~m}^{3} / \mathrm{h}$ at $35^{\circ} \mathrm{C}$.

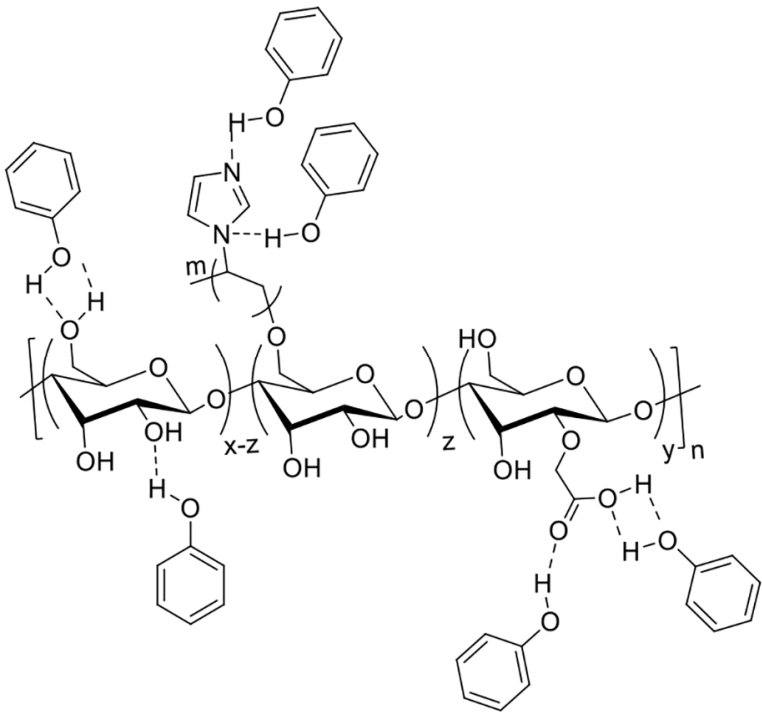

conical flasks (e) connected to sample glass tube containing adsorbent from the concentration of phenol in the conical flasks (e) attached to the blank sample glass tube.

The results for phenol adsorption experiment are depicted in Fig. 7. The adsorption affinities for CMS, CMS-g-PVI 1, CMS-g-PVI 2 and CMS-g-PVI 3 were found to be 0.170, 0.190, 0.192 and 0.199 g/g.

The adsorption of phenol by CMS-g-PVIs can be explained in four different ways. (i) As the CMS has bunch of $-\mathrm{OH}$ groups on its backbone, which make hydrogen bonding with - $\mathrm{OH}$ group on phenol. So, in this way the modified starch adsorb phenol, the adsorption mechanism is physi-sorption. (ii) The CMS also has $-\mathrm{COOH}$ groups on its main chain. These $-\mathrm{COOH}$ groups are also capable to make hydrogen bonding. In this case the hydrogen bonding is generated between - $\mathrm{OH}$ group of phenol and $-\mathrm{COOH}$ groups present on CMS main chain. (iii) The grafted chains of PVI contain imidazole ring in its structure. The imidazole ring contains nitrogen atoms may act as hydrogen bond acceptor. So, these nitrogen atoms make hydrogen bonding with the - $\mathrm{OH}$ group of phenol. (iv) The $\pi-\pi$ interactions can be generated between the aromatic benzene ring in phenol and aromatic imidazole ring of PVI [31]. The suggested phenol adsorption mechanism is sketched in Fig. 8.

\section{Conclusion}

Cigarette smoke contains many toxic chemicals, which directly affect human life. In this research, CMS-g-PVIs were produced by reacting CMS with vinyl imidazole. The structures of CMS-g-PVIs were confirmed by FT-IR and ${ }^{1} \mathrm{H}$ NMR. After modification the CMS-g-PVIs showed amorphous nature and enhanced thermal properties due to introduction of aromatic imidazole ring. The CMS-g-PVIs were subjected for phenol adsorption. The obtained results showed adsorption efficiencies for phenol $0.170 \mathrm{~g} / \mathrm{g}, 0.190$

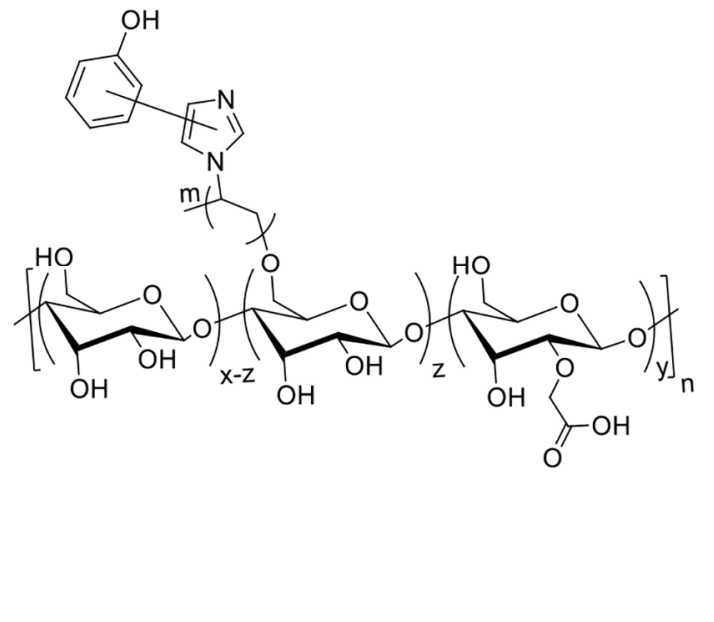

Fig. 8. Possible adsorption mechanism based on (a) hydrogen bonding and (b) $\pi-\pi$ stacking. 
g/g, $0.192 \mathrm{~g} / \mathrm{g}$ and $0.199 \mathrm{~g} / \mathrm{g}$ by CMS, CMS-g-PVI 1, CMS-g-PVI 2 and CMS-g-PVI 3. The results showed that CMS-g-PVIs are effective to adsorb phenol from cigarette smoke.

\section{Author Contributions}

F.H. (Ph.D) conducted the research and investigation process and wrote the original draft. H.Y (Associate Professor) revised the topic several times and provided his suggestions and gave the final approval of the last version to be submitted. L.W. (Professor) revised the final version and provided his comments focusing on the synthetic routes. L.T. (Professor) revised the topic from the beginning to the end providing his suggestions about the contents and the format. S.M. (Ph.D) participated in jotting down the application section. M.H. (Assistant Professor) helped to design the reaction parameters and conditions by providing in depth analysis. B.U.A. (Ph.D) checked the references styles and abbreviations. M.A.U. (Ph.D) edited the contents and checked the similarity. S.D. (Ph.D) revised the grammatical issues and the coherence of the text.

\section{References}

1. John E, Coburn S, Liu C, et al. Effect of temperature and humidity on the gas-particle partitioning of nicotine in mainstream cigarette smoke: A diffusion denuder study. J. Aerosol. Sci. 2018;117:100-117.

2. Branton P, Lu AH, Schüth F. The effect of carbon pore structure on the adsorption of cigarette smoke vapour phase compounds. Carbon 2009;47:1005-1011.

3. Mendel JR, Baig SA, Hall MG, et al. Brand switching and toxic chemicals in cigarette smoke: A national study. PloS One. 2018;13:e0189928.

4. Noar SM, Kelley DE, Boynton MH, et al. Identifying principles for effective messages about chemicals in cigarette smoke. Prev. Med. 2018;106:31-37.

5. Berlin I, Hakes JK, Hu MC, Covey LS. Identifying principles for effective messages about chemicals in cigarette smoke. Plos One. 2015;10:e0122607.

6. Boden JM, Fergusson DM, Horwood LJ. Cigarette smoking and suicidal behaviour: results from a 25-year longitudinal study. Psychol. Med. 2008;38:433-439.

7. Bohnert KM, Ilgen MA, McCarthy JF, Ignacio RV, Blow FC, Katz IR. Tobacco use disorder and the risk of suicide mortality. Addiction 2014;109:155-162.

8. Korhonen T, Sihvola E, Latvala A, et al. Early-onset tobacco use and suicide-related behavior-A prospective study from adolescence to young adulthood. Addict. Behav. 2018;79:32-38.

9. Miller M, Hemenway D, Bell NS, Yore MM, Amoroso PJ. Cigarette smoking and suicide: A prospective study of 300, 000 male active-duty army soldiers. Am. J. Epidemiol. 2000;151:1060-1063.

10. Miller M, Hemenway D, Rimm E. Cigarettes and suicide: A prospective study of 50,000 men. Am. J. Public Health. 2000;90: 768-773.

11. Poorolajal J, Darvishi N. Smoking and suicide: A meta-analysis. PloS One. 2016;11:e0156348.
12. Ahmaruzzaman M. Adsorption of phenolic compounds on low-cost adsorbents: A review. Adv. Colloid. Interface. Sci. 2008;143:48-67.

13. Chortyk OT, Schlotzhauer WS. The contribution of low tar cigarettes to environmental tobacco smoke. J. Anal. Toxicol. 1989;13:129-134.

14. Michałowicz J, Duda W. Phenols-sources and toxicity. Pol. J. Environ.Stud. 2007;16:347-362.

15. Pistonesi MF, Di Nezio MS, Centurión ME, et al. Simultaneous determination of hydroquinone, resorcinol, phenol, m-cresol and p-cresol in untreated air samples using spectrofluorimetry and a custom multiple linear regression-successive projection algorithm. Talanta 2010;83:320-323.

16. Chen H, Wang C, Ye J, Zhou H, Tao R, Li W. Preparation of starch-hard carbon spherules from ginkgo seeds and their phenol-adsorption characteristics. Molecules 2018;23:96.

17. Denizli A, Cihangir N, Tüzmen N, Alsancak G. Removal of chlorophenols from aquatic systems using the dried and dead fungus Pleurotus sajor caju. Bioresour. Technol. 2005;96:59-62.

18. Termoul M, Bestani B, Benderdouche N, Belhakem M, Naffrechoux E. Removal of phenol and 4-chlorophenol from aqueous solutions by olive stone-based activated carbon. Adsorpt. Sci. Technol. 2006;24:375-388.

19. Aksu Z, Yener J. Investigation of the biosorption of phenol and monochlorinated phenols on the dried activated sludge. Process. Biochem. 1998; 33:649-655.

20. Pour ZS, Ghaem M. Removal of dyes and heavy metal ions from water by magnetic hydrogel beads based on poly(vinyl alcohol)/carboxymethyl starch-g-poly(vinyl imidazole). RSC. Adv. 2015;5:64106-64118.

21. El-Hamshary H, Fouda MMG, Moydeen M, Al-Deyab SS. Removal of heavy metal using poly ( $N$-vinylimidazole)- grafted-carboxymethylated starch. Int. J. Biol. Macromol. 2014;66: 289-294.

22. Kutcherlapati SR, Koyilapu R, Jana T. Poly(N-vinyl imidazole) grafted silica nanofillers: Synthesis by RAFT polymerization and nanocomposites with polybenzimidazole. J. Polym. Sci. Pol. Chem. 2018;56:365-375.

23. Fodor C, Bozi J, Bazso M, Ivan B. Thermal behavior, stability, and decomposition mechanism of poly( $N$-vinylimidazole). Macromolecules 2012;45:8953-8960.

24. Haroon M, Yu H, Wang L, Ullah RS, Haq F, Teng L. Synthesis and characterization of carboxymethyl starch-g-polyacrylic acids and their properties as adsorbents for ammonia and phenol. Int. J. Biol. Macromol. 2019;138:349-358.

25. Pekel N, Güven O. Separation of uranyl ions with amidoximated poly(acrylonitrile/ $N$-vinylimidazole) complexing sorbents. Colloid. Surf. A- Physicochem. Eng. Asp. 2003;212:155-161.

26. Ramasamy R. Vibrational spectroscopic studies of imidazole. Armen. J. Phys. 2015;8:51-55.

27. Liu J, Ming J, Li W, Zhao G. Synthesis, characterisation and in vitro digestibility of carboxymethyl potato starch rapidly prepared with microwave-assistance. Food Chem. 2012;133: 1196-1205.

28. Sabaa MW, Magid EHA, Mohamed RR. Maize starch-g-poly (n-vinylimidazole) Synthesis and its application in sewage water treatment. Research and reviews: J. Chem. 2017;6:55-67. 
29. Chen Q, Yu H, Wang L, et al. Synthesis and characterization of amylose grafted poly (acrylic acid) and its application in ammonia adsorption. Carbohydr. Polym. 2016;153:429-434.

30. Pekel N, Güven O. Synthesis and characterization of poly( $N$-vinyl imidazole) hydrogels crosslinked by gamma irradiation.
Polym. Int. 2002;51:1404-1410.

31. Abdelhamid R, Obara Y, Uchida Y, et al. Pi-pi interaction between aromatic ring and copper-coordinated His81 imidazole regulates the blue copper active-site structure. J. Biol. Inorg. Chem. 2007;12:165-173. 